\title{
STATIONARY POLICIES FOR THE SECOND MOMENT STABILITY IN A CLASS OF STOCHASTIC SYSTEMS
}

\author{
Alessandro N. Vargas and João B. R. do Val
}

\begin{abstract}
This paper presents a study on the uniform second moment stability for a class of stochastic control system. The main result states that the existence of the long-run average cost under a stationary policy is equivalent to the uniform second moment stability of the corresponding stochastic control system. To illustrate the result, a numerical example is developed to verify the uniform second moment stability of a simultaneous state-feedback control system.

Index Terms - average cost problems, second moment stability, stochastic control, Markov processes.
\end{abstract}

\section{INTRODUCTION}

Consider a time-varying discrete-time stochastic linear system defined in a filtered probability space $\left(\Omega, \mathcal{F},\left\{\mathcal{F}_{k}\right\}, P\right)$ as follows.

$$
x_{k+1}=A_{k} x_{k}+E_{k} w_{k}, \quad y_{k}=C_{k} x_{k}, \quad \forall k \geq 0, \quad x_{0} \in \mathbb{R}^{n},
$$

where $x_{k}, y_{k}$, and $w_{k}, k=0,1, \ldots$ are processes taking values respectively, in $\mathbb{R}^{n}, \mathbb{R}^{p}$ and $\mathbb{R}^{q}$, which represent the system state, output, and additive noisy input, respectively. The noisy input $\left\{w_{k}\right\}$ forms an iid process with zero mean and covariance matrix equal to the identity for all $k \geq 0$. The matrices $A_{k}, C_{k}$, and $E_{k}$ are given, and they have dimensions $n \times n, p \times n$ and $n \times q$, respectively. Let us associate the time-varying system (1) with the long-run average cost

$$
J=\limsup _{N \rightarrow \infty} \frac{1}{N} \sum_{k=0}^{N-1} \mathrm{E}\left[\left\|y_{k}\right\|^{2}\right],
$$

where $\mathrm{E}[\cdot] \equiv \mathrm{E}\left[\cdot \mid x_{0}\right]$ denotes the mathematical expectation and $\|\cdot\|$ the usual Euclidean norm.

The main contribution of this paper can be seen as an advance with respect to [1] regarding the characterization of uniform second moment stability for the system (1). Indeed, the authors in [1] show that, if $J$ is finite, then the time-varying system (1) is asymptotically stable in

Research supported in part by Brazilian agencies FAPESP Grant 03/06736-7 and CNPq Grants 471557/2009 and 304856/2007-0, and in part by Spanish agency Fundación Carolina - Programa "Movilidad de profesores e investigadores Brasil-España. C.2010".

Alessandro N. Vargas is with Universidade Tecnológica Federal do Paraná, UTFPR, Av. Alberto Carazzai 1640, 86300-000 Cornelio Procópio-PR, Brazil, and with Basque Center for Applied Mathematics, BCAM, Bizkaia Technology Park, Building 500, E48160 Derio, Basque Country, Spain (from January 1, 2011 to February 28, 2011). E-mail: avargas@utfpr.edu.br

João B. R. do Val is with Universidade Estadual de Campinas, UNICAMP, DT/FEEC, C.P. 6101, 13081-970 Campinas-SP, Brazil. E-mail: jbosco@dt.fee.unicamp.br the mean, i.e., $\mathrm{E}\left[x_{k}\right] \rightarrow 0$ as $k \rightarrow \infty[1$, Cor. 3.1$]$, provided that the pairs $\left(A_{k}, E_{k}\right)$ and $\left(A_{k}, C_{k}\right)$ are controllable and observable, respectively. However, the stronger concept of stability, known as uniform second moment stability, is not studied in details in [1]. By considering this gap in the literature, our approach aims to complement the investigation by providing conditions to assure the second moment stability of (2). Namely, from the existence of the average cost $J$ in (2), we particularize the matrices $A_{k}$ and $C_{k}$ to assure the second moment stability for (2).

In this paper, the matrices $A_{k}$ and $C_{k}$ are made up according to a particular rule of construction, as follows. Let us consider a prescribed set $\mathcal{G}$, which is called control set, and let the variable $g_{k} \in \mathcal{G}$, at the $k$-th stage, represents the control action. We now assume that $A$ : $\mathcal{G} \mapsto \mathbb{R}^{n \times n}$ represents a continuous operator, possibly a nonlinear one; and $Q: \mathcal{G} \mapsto \mathbb{S}_{+}^{n}$ represents an arbitrary operator, where $\mathbb{S}_{+}^{n}$ denotes the space of nonnegative symmetric matrices of dimension $n \times n$. In (1) and (2), the specialization is obtained by setting

$$
A_{k} \equiv A\left(g_{k}\right) \quad \text { and } \quad C_{k}^{\prime} C_{k} \equiv Q\left(g_{k}\right) .
$$

Let us define the second moment of the system state $x_{k}$ as

$$
X_{k}=\mathrm{E}\left[x_{k} x_{k}^{\prime}\right], \quad \forall k \geq 0 .
$$

The control action $g_{k} \in \mathcal{G}$ applied in (1)-(3) is assumed to be a function of the second moment only, i.e., it takes the deterministic feedback form $g_{k}=f_{k}\left(X_{k}\right)$ for each $k \geq 0$. This special form suggests simplicity of solutions, since it turns valid the identity

$$
\mathrm{E}\left[x_{k}^{\prime} Q\left(g_{k}\right) x_{k}\right]=\left\langle Q\left(g_{k}\right), X_{k}\right\rangle,
$$

where $\langle\cdot, \cdot\rangle$ represents the usual Frobenius inner product. The control action in the form $g_{k}=f_{k}\left(X_{k}\right)$ is useful (see [2], [3], and [4]), specially when $g_{k}$ is a gain matrix, see Section II-A for an application in the control problem of the simultaneous state-feedback system.

We focus our investigation on the second moment stability of the system (1) and (3) with control actions in the form $g_{k}=f\left(X_{k}\right), k \geq 0$, or equivalently under a stationary policy $\mathbf{f}_{s}=\{f, f, \ldots\}$. The main result shows that the system (1) and (3) is uniformly second moment stable under a stationary policy $\mathbf{f}_{s}=\{f, f, \ldots\}$ if and only if the corresponding long-run average cost in (2) is finite, see Theorem 2.1 in connection.

An additional motivation for the current investigation is that stationary policies producing a finite long-run 
average cost is an important topic in optimal control systems, see [2], [3], [4]. Thus, our result can be used to guarantee the second moment stability of the optimal solution for control problems that can be rewritten in the form (1)-(3).

The paper is organized as follows. Section II presents the necessary notation, definitions, assumptions, and the main result. The main result concerning the uniform second moment stability is presented in Theorem 2.1. To illustrate the result, we introduce an application to the simultaneous state-feedback control systems in Section II-A.

\section{Preliminaries, notations, and main Results}

The real and natural numbers are denoted by $\mathbb{R}$ and $\mathbb{N}$, respectively. The set of nonnegative real numbers is denoted by $\mathbb{R}_{+}$, and $\mathbb{R}^{n, m}$ is used to represent the space of all $n \times m$ real matrices. The superscript ' indicates the transpose of a matrix. Let $\mathbb{S}_{+}^{n}$ be the closed convex cone $\left\{U \in \mathbb{R}^{n, n}: U=U^{\prime} \geq 0\right\} ;\langle\cdot, \cdot\rangle$ will stand the Frobenius inner product in $\mathbb{S}_{+}^{n}$, and $\|\cdot\|$ will denote either the standard Euclidean norm in $\mathbb{R}^{n}$ or the Frobenius norm for matrices. Let $\operatorname{tr}\{\cdot\}$ be the trace operator. We recall that $\|U\|^{2}=\operatorname{tr}\left\{U^{2}\right\} \leq \operatorname{tr}\{U\}^{2}$ whenever $U \in \mathbb{S}_{+}^{n}$, see [5]. We say that a matrix sequence $\left\{U_{k} ; k \geq 0\right\}$ is bounded if $\sup _{k \in \mathbb{N}}\left\|U_{k}\right\|<\infty$.

For sake of simplicity, let us rewrite the system (1)-(3) equivalently as

$$
x_{k+1}=A\left(g_{k}\right) x_{k}+E_{k} w_{k}, \quad g_{k} \in \mathcal{G}, \quad \forall k, \quad x_{0} \in \mathbb{R}^{n},
$$

and the corresponding long-run average cost as

$$
J=\limsup _{N \rightarrow \infty} \frac{1}{N} \sum_{k=0}^{N-1} \mathrm{E}\left[x_{k}^{\prime} Q\left(g_{k}\right) x_{k}\right]
$$

The following definitions and conventions will apply throughout this paper.

(i) $\mathcal{X}$ and $\mathcal{G}$ are given sets referred to as state space and control space, respectively. In particular, we assume that $\mathcal{X}$ is a subset of $\mathbb{S}_{+}^{n}$ and $\mathcal{G}$ is a given Borel set.

(ii) Let $Q: \mathcal{G} \rightarrow \mathbb{S}_{+}^{n}$ be a given continuous function such that $Q(g)>0$ for each $g \in \mathcal{G}$. The one-stage cost functional $\mathcal{e}: \mathcal{X} \times \mathcal{G} \mapsto \mathbb{R}_{+}$is defined as follows:

$$
\mathcal{C}(X, g)=\langle X, Q(g)\rangle, \quad \forall X \in X, \forall g \in \mathcal{G} .
$$

(iii) (inf-compactness [6, p.28]). For each $X \in X$ and $\lambda \in$ $\mathbb{R}_{+}$, the set of control actions $\{g \in \mathcal{G}: \mathcal{C}(X, g) \leq \lambda\}$ is compact.

(iv) The measurable function $f: \mathcal{X} \rightarrow \mathcal{G}$ is called control law whenever $f(X) \in \mathcal{G}$ for each $X \in X$. A policy $\mathbf{f}=\left\{f_{0}, f_{1}, \ldots\right\}$ is a sequence of control laws $f_{k}, k \geq 0$, and the set of all policies is denoted by $\mathbf{F}$. Elements of $\mathbf{F}$ of the form $\mathbf{f}_{s}=\{f, f, \ldots\}$ are referred to as stationary policies.

From the assumption on the process $\left\{w_{k}\right\}, k \geq 0$, and for a given policy $\mathbf{f}=\left\{f_{k}\right\} \in \mathbf{F}$, the second moment matrix
$X_{k} \in X$ from (4) satisfies the recurrence (c.f. [7, Ch.2])

$$
X_{k+1}=A\left(g_{k}\right) X_{k} A\left(g_{k}\right)^{\prime}+\Sigma_{k}, \quad \forall k \geq 0, \quad \forall X_{0}=X \in X,
$$

with $\Sigma_{k}:=E_{k} E_{k}^{\prime}$, where the control obeys the rule

$$
g_{k}=f_{k}\left(X_{k}\right), \quad \forall k \geq 0 .
$$

Sometimes we use the notation $X_{k}^{(\mathbf{f})}$ to stress that the recurrence (8) depends on a specific $\mathbf{f} \in \mathbf{F}$. Moreover, we represent the $k$-th stage cost by

$$
\mathcal{C}_{k}^{(\mathbf{f}, X)}:=\mathcal{C}\left(X_{k}^{(\mathbf{f})}, g_{k}\right)=\left\langle X_{k}^{(\mathbf{f})}, Q\left(g_{k}\right)\right\rangle, \quad \forall k \geq 0,
$$

and so the corresponding long-run average cost is given by

$$
J(\mathbf{f}, X):=\limsup _{N \rightarrow \infty} \frac{1}{N} \sum_{k=0}^{N-1} \mathrm{e}_{k}^{(\mathbf{f}, X)}, \quad \forall \mathbf{f} \in \mathbf{F}, \forall X \in X .
$$

Given $\mathbf{f}=\left\{f_{k}\right\} \in \mathbf{F}$, let us define a family of discrete evolution operators associated with (8) and (9) as

$$
\Phi(k, m)=A\left(g_{k-1}\right) A\left(g_{k-2}\right) \cdots A\left(g_{m}\right), \quad \forall k>m \geq 0,
$$

with $\Phi(m, m)=I$ and $g_{k} \in \mathcal{G}, k \geq 0$, as in (9). These evolution operators allow us to write

$$
X_{k}=\Phi(k, m) X_{m} \Phi(k, m)^{\prime}+\sum_{j=m}^{k-1} \Phi(k, j+1) \Sigma_{j} \Phi(k, j+1)^{\prime}
$$

for each $0 \leq m \leq k$, with $X_{m} \in \mathbb{S}_{+}^{n}$.

The next definition introduces a notion of observability for the system (5)-(6).

Definition 2.1: The system (5)-(6) is uniformly observable under $\mathbf{f}=\left\{f_{k}\right\} \in \mathbf{F}$ if there exist $T_{o} \geq 1$ and $\beta>0$ such that, for each $k \geq 0$,

$$
\sum_{i=0}^{T_{o}-1} \Phi(k+i, k)^{\prime} Q\left(g_{k+i}\right) \Phi(k+i, k) \geq \beta I,
$$

where $g_{k} \in \mathcal{G}, k \geq 0$, satisfies (9).

Proposition 2.1: ([1, Lem. 2.3]). If the system (5)-(6) is uniformly observable under $\mathbf{f}=\left\{f_{k}\right\} \in \mathbf{F}$, then there exist $T_{o} \geq 1$ and $\mu>0$ such that

$$
\sum_{i=k}^{n+T_{o}} \mathrm{C}_{i}^{(\mathbf{f}, X)} \geq \mu \sum_{i=k}^{n} \operatorname{tr}\left\{X_{i}\right\}, \quad \forall n \geq k \in \mathbb{N}
$$

where $X_{k}, k \geq 0$, satisfies (8)-(9) with $X_{0}=X \in X$.

Now, we introduce the definition of uniform second moment stability for the system (5).

Definition 2.2: [8, p.97-98]. The stochastic system (5) is called uniformly second moment stable under $\mathbf{f}=$ $\left\{f_{k}\right\} \in \mathbf{F}$ if there exists a constant $c>0$ such that

$$
\mathrm{E}\left[\left\|x_{k}\right\|^{2}\right] \leq c, \quad \forall k \geq 0,
$$

where $x_{k} \in \mathbb{R}^{n}$ satisfies (5) with $g_{k} \in \mathcal{G}$ as in (9).

Remark 2.1: From the definition in (4), we get the identity [7, Ch.2]

$$
\mathrm{E}\left[\left\|x_{k}\right\|^{2}\right]=\operatorname{tr}\left\{X_{k}\right\}, \quad \forall k \geq 0,
$$


where $X_{k}, k \geq 0$, satisfies (8)-(9). Since the inequality $\operatorname{tr}\left\{X_{k}\right\} \geq\left\|X_{k}\right\|$ is valid for each $k \geq 0$, one can see that the system (5) is uniformly second moment stable under $\mathbf{f}=\left\{f_{k}\right\} \in \mathbf{F}$ if and only if the matrix sequence $\left\{X_{k}\right\}$ is bounded.

Hereafter, we restrict our analysis to stationary policies, i.e., those of the form $\mathbf{f}_{s}=\{f, f, \ldots\}$ in order to characterize the second moment stability of (5).

The next theorem presents the main result of this paper.

Theorem 2.1: Let $f$ be some control function such that (5)-(6) is uniformly observable under the stationary policy $\mathbf{f}_{s}=\{f, f, \ldots\}$. If the sequences $\left\{A\left(g_{k}\right)\right\}$ and $\left\{Q\left(g_{k}\right)\right\}$ are bounded, with $g_{k}=f\left(X_{k}\right)$ and $X_{k}$ satisfying (8), then the following two assertions are equivalent:

(i) The system (5) is uniformly second moment stable under the stationary policy $\mathbf{f}_{s}=\{f, f, \ldots\}$.

(ii) There exists a constant $\rho>0$ such that $J\left(\mathbf{f}_{s}, X\right) \leq \rho$ for all $X \in X$.

Proof: $[(\mathrm{i}) \Rightarrow(\mathrm{ii})]$. The uniform second moment stability implies that the matrix sequence $\left\{X_{k}\right\}$ is bounded (see Remark 2.1), and from the assumption that $\left\{Q\left(g_{k}\right)\right\}$ is bounded, we have that there is a constant $\rho>0$ such that

$$
\mathcal{C}_{k}^{\left(\mathbf{f}_{s}, X\right)}=\left\langle X_{k}, Q\left(g_{k}\right)\right\rangle \leq \rho, \quad k \geq 0 .
$$

Hence, $J\left(\mathbf{f}_{s}, X\right) \leq \rho$ for all $X \in \mathcal{X}$.

$\left[(\right.$ i $) \Leftarrow$ (ii)]. Recall that $\operatorname{tr}\{U\} \geq\|U\|$ whenever $U \in \mathbb{S}_{+}^{n}$. Combining this inequality and Proposition 2.1, we have that there are constants $T_{o} \geq 1$ and $\mu>0$ such that

$$
\sum_{i=k}^{n+T_{o}} \mathcal{C}_{i}^{\left(\mathbf{f}_{s}, X\right)} \geq \mu \sum_{i=k}^{n} \operatorname{tr}\left\{X_{i}\right\} \geq \mu \sum_{i=k}^{n}\left\|X_{i}\right\|, \quad \forall n \geq k \in \mathbb{N} .
$$

We now prove that the matrix sequence $\left\{X_{k}\right\}$ does not diverge to infinity. Using a contradiction argument, let us assume that $\left\{X_{k}\right\}$ diverges to infinity. In this case

$$
\frac{1}{N+T_{o}} \sum_{k=0}^{N+T_{o}-1} \mathcal{C}_{k}^{\left(\mathbf{f}_{s}, X\right)} \geq \frac{\mu}{N+T_{o}} \sum_{k=0}^{N-1}\left\|X_{k}\right\| \rightarrow \infty \quad \text { as } \quad N \rightarrow \infty,
$$

which yields $J\left(\mathbf{f}_{s}, X\right)=\infty$ and so the contradiction arises.

Hence $\left\{X_{k}\right\}$ does not diverge to infinity, or equivalently the sequence $\left\{\left\|X_{k}\right\|\right\}$ has at least one subsequence which is bounded by a sufficiently large constant $M$. Namely, there is a sequence $\left\{t_{k}\right\}$ from $\mathbb{N}$ such that

$$
\left\|X_{t_{k}}\right\| \leq M, \quad \forall k \geq 0 .
$$

For sake of convenience in the proof of the next argument, we assume that $M>(\rho+1) / \mu$.

We claim that there is a constant $\delta \geq 1$ such that

$$
s_{k}:=t_{k+1}-t_{k} \leq \delta .
$$

The result of this claim is crucial, since it assures that the trajectory $\left\{X_{k}\right\}$ is bounded. Indeed, notice from (13) that

$$
\begin{aligned}
X_{t_{k}+m}= & \Phi\left(t_{k}+m, t_{k}\right) X_{t_{k}} \Phi\left(t_{k}+m, t_{k}\right)^{\prime} \\
& +\sum_{j=t_{k}}^{t_{k}+m-1} \Phi\left(t_{k}+m, j+1\right) \Sigma_{j} \Phi\left(t_{k}+m, j+1\right)^{\prime} \\
\leq & a^{2 m} M+\lambda \sum_{j=0}^{m} a^{2 j}, \quad m=0,1, \ldots, \delta,
\end{aligned}
$$

where $a:=\sup _{k \in \mathbb{N}}\left\|A\left(g_{k}\right)\right\|$ and $\lambda:=\sup _{k \in \mathbb{N}}\left\|\Sigma_{k}\right\|$. Under the claim that $\delta$ is finite, the upper bound $a^{2 \delta} M+$ $\lambda \sum_{j=0}^{\delta} a^{2 j}$ is also finite and so the sequence $\left\{\left\|X_{k}\right\|\right\}$ is bounded. This argument proves the result under the assumption that $\delta$ is finite.

In order to prove that $\delta$ is finite, we use a contradiction argument as follows. Let us consider $s_{k}$ as in (17) with a subsequence $\left\{t_{k_{n}}\right\}$ from $\left\{t_{k}\right\}$ such that $s_{k_{n}} \rightarrow \infty$ as $n \rightarrow \infty$. Note that (16) assures the inequality $\left\|X_{m}\right\|>M$ when $m \neq t_{k}, \forall k \geq 0$. Hence we can use (15) to write

$$
\begin{aligned}
\sum_{i=0}^{T_{o}+s_{k_{n}}-1} \mathcal{C}_{i}^{\left(\mathbf{f}_{s}, X_{t_{k_{n}}}\right)} & =\sum_{i=0}^{T_{o}+s_{k_{n}}-1} \mathcal{e}_{t_{k_{n}}+i}^{\left(\mathbf{f}_{s}, X\right)} \\
& =\sum_{i=0}^{T_{o}+s_{k_{n}}-1}\left\langle X_{t_{k_{n}}+i}, Q\left(g_{t_{k_{n}}+i}\right)\right\rangle \\
& \geq \sum_{i=1}^{s_{k_{n}}-1} \mu\left\|X_{t_{k_{n}}+i}\right\|>\left(s_{k_{n}}-1\right) \mu M
\end{aligned}
$$

Dividing both sides of (18) by $\left(T_{o}+s_{k_{n}}\right)$, and recalling that $M>(\rho+1) / \mu$, we obtain

$$
\begin{aligned}
\frac{1}{T_{o}+s_{k_{n}}} \sum_{i=0}^{T_{o}+s_{k_{n}}-1} \mathcal{C}_{i}^{\left(\mathbf{f}_{s}, X_{k_{k_{n}}}\right)} & \\
& >\frac{\left(s_{k_{n}}-1\right)}{T_{o}+s_{k_{n}}}(\rho+1)=\frac{\left(1-\frac{1}{s_{k_{n}}}\right)}{\frac{T_{o}}{s_{k_{n}}}+1}(\rho+1) .
\end{aligned}
$$

Since $s_{k_{n}} \rightarrow \infty$ as $n \rightarrow \infty$, we can conclude that there exists a sufficiently large number $n_{0} \in \mathbb{N}$ such that

$$
n \geq n_{0} \Rightarrow \frac{1}{T_{o}+s_{k_{n}}} \sum_{i=0}^{T_{o}+s_{k_{n}}-1} \mathcal{C}_{i}^{\left(\mathbf{f}_{s}, X_{t_{k_{n}}}\right)}>\rho \geq J\left(\mathbf{f}_{s}, X_{t_{k_{n}}}\right),
$$

which is an absurd. This argument shows that $\delta$ in (17) is finite and so we have just proved that there is a constant $c>0$, which may depend on $\rho$, such that $\left\|X_{k}\right\| \leq c$ for all $k \geq 0$. The argument in Remark 2.1 then guarantees that the system (5) is uniformly second moment stable under $\mathbf{f}_{s}=\{f, f, \ldots\}$.

\section{A. Application to the simultaneous state-feedback prob-} lem

The simultaneous state-feedback control system can be represented as (see [9], [10], [11], [12], [13], and [14] for a small account)

$$
\begin{array}{r}
\varphi_{i}(k+1)=\left(A_{i}+B_{i} g(k)\right) \varphi_{i}(k)+H_{i} \omega_{i}(k), \quad \varphi_{i}(0) \in \mathbb{R}^{n} \\
i=1, \ldots, \sigma,
\end{array}
$$


where $g(k)$ is a design gain matrix that does not depend on the mode $i$, and $\varphi_{i}(\cdot)$ and $\omega_{i}(\cdot)$ represent the simultaneous system state and additive noise input for the $i$-th mode, respectively. The long-run average cost associated with (19) is given by

$$
\begin{aligned}
J(\varphi(0))=\limsup _{N \rightarrow \infty} \frac{1}{N} \sum_{k=0}^{N-1}( & \sum_{i=1}^{\sigma} \mathrm{E}\left[\varphi _ { i } ( k ) ^ { \prime } \left(Q_{i}\right.\right. \\
& \left.\left.\left.\left.+g(k)^{\prime} R_{i} g(k)\right) \varphi_{i}(k)\right)\right]\right),
\end{aligned}
$$

where the matrices $Q_{i}=Q_{i}^{\prime} \geq 0$ and $R_{i}=R_{i}^{\prime}>0$ are given.

We claim that (19)-(20) is a particular case of (5)-(6). Indeed, by considering the matrix dimensions

$$
\operatorname{dim}\left(A_{i}\right)=n \times n, \operatorname{dim}\left(B_{i}\right)=n \times r, \text { and } \operatorname{dim}\left(H_{i}\right)=n \times q,
$$

and by setting $\mathcal{G} \equiv \mathbb{R}^{r \times n}$, we can define the operators

$$
\begin{aligned}
& A(g)=\operatorname{diag}\left(A_{1}+B_{1} g, \ldots, A_{\sigma}+B_{\sigma} g\right), \quad \forall g \in \mathcal{G}, \\
& Q(g)=\operatorname{diag}\left(Q_{1}+g^{\prime} R_{1} g, \ldots, Q_{\sigma}+g^{\prime} R_{\sigma} g\right), \quad \forall g \in \mathcal{G} .
\end{aligned}
$$

Moreover, take $E_{k} \equiv \operatorname{diag}\left(H_{1}, \ldots, H_{\sigma}\right)$ in (5). Thus, the correspondence between (5)-(6) and (19)-(20) follows by stacking both the state and the additive noise input of the simultaneous system, respectively, in the format

$$
x_{k}=\left[\begin{array}{c}
\varphi_{1}(k) \\
\vdots \\
\varphi_{\sigma}(k)
\end{array}\right] \in \mathbb{R}^{\sigma n}, \quad \text { and } \quad w_{k}=\left[\begin{array}{c}
\omega_{1}(k) \\
\vdots \\
\omega_{\sigma}(k)
\end{array}\right] \in \mathbb{R}^{\sigma q} .
$$

We can conclude from the above discussion that the simultaneous system (19)-(20) is a particular case of (5)(6). This fact enables us to apply the stability result of Theorem 2.1 for the simultaneous system as follows.

We are interested in determining a control law $f$, or equivalently a stationary policy $\mathbf{f}_{s}=\{f, f, \ldots\}$, such that the simultaneous system (19)-(20) is uniformly second moment stable under $\mathbf{f}_{s}=\{f, f, \ldots\}$. For this purpose, let us define

$$
\Psi_{i}(k):=\mathrm{E}\left[\varphi_{i}(k) \varphi_{i}(k)^{\prime}\right], \quad \forall k \geq 0, i=1, \ldots, \sigma .
$$

The following recurrence is valid [7, Ch.2]:

$$
\begin{array}{r}
\Psi_{i}(k+1)=\left(A_{i}+B_{i} g(k)\right) \Psi_{i}(k)\left(A_{i}+B_{i} g(k)\right)^{\prime}+H_{i} H_{i}^{\prime}, \\
\forall k \geq 0, \quad i=1, \ldots, \sigma .
\end{array}
$$

Let $L_{i} \in \mathbb{S}_{+}^{n}, i=1, \ldots, \sigma$, be the solution of the Riccati equation

$L_{i}=Q_{i}+A_{i}^{\prime} L_{i} A_{i}-A_{i}^{\prime} L_{i} B_{i}\left(R_{i}+B_{i}^{\prime} L_{i} B_{i}\right)^{-1} B_{i}^{\prime} L_{i} A_{i}, i=1, \ldots, \sigma$, and let $K_{i} \in \mathbb{R}^{r \times n}, i=1, \ldots, \sigma$, be the corresponding Kalman gain

$$
K_{i}=\left(R_{i}+B_{i}^{\prime} L_{i} B_{i}\right)^{-1} B_{i}^{\prime} L_{i} A_{i}, i=1, \ldots, \sigma .
$$

For a given $U=\left(U_{1}, \ldots, U_{\sigma}\right)$, where each $U_{i}, i=1, \ldots, \sigma$, belongs to $\mathbb{S}_{+}^{n}$, we define

$$
i^{*}(U)=\underset{i=1, \ldots, \sigma}{\arg \max }\left\langle L_{i}, U_{i}^{2}\right\rangle .
$$
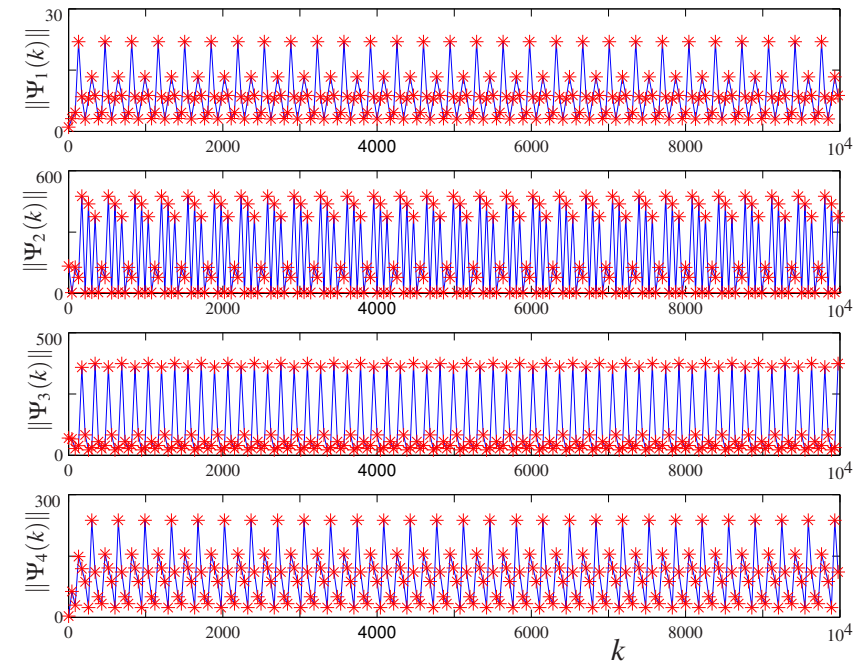

Fig. 1. The norm of the trajectory $\Psi(k), k \geq 0$, with initial condition $\varphi_{i}(0)=\left[\begin{array}{lll}0.48 & 0.89 & 0.76\end{array}\right]^{\prime}, i=1, \ldots, 4$, according to the numerical example of Section II-A.

By setting $\Psi(k)=\left(\Psi_{1}(k), \ldots, \Psi_{\sigma}(k)\right)$ for each $k \geq 0$, we can define the control function

$$
f(\Psi(k))=K_{i^{*}(\Psi(k))}, \quad \forall k \geq 0,
$$

and also the corresponding control action $g(k), k \geq 0$, within (19)-(20) as follows:

$$
g(k)=f(\Psi(k)), \quad \forall k \geq 0 .
$$

Note that $f$ defined in (25) sets the stationary policy $\mathbf{f}_{s}=\{f, f, \ldots\}$.

Now, we present a numerical example to illustrate the result of Theorem 2.1. Let us consider the simultaneous system in (19) with four different operating points

$$
A_{i}=\left[\begin{array}{ccc}
a_{11}^{i} & a_{12}^{i} & a_{13}^{i} \\
a_{21}^{i} & a_{22}^{i} & a_{23}^{i} \\
0 & 0 & 0.2231
\end{array}\right], B_{i}=\left[\begin{array}{c}
b_{1}^{i} \\
b_{2}^{i} \\
0.7769
\end{array}\right], i=1, \ldots, 4,
$$

where the parameters $a_{i j}^{i}$ and $b_{i}^{i}$ are as listed in [15]. We adopt $H_{i}=0.5 I, Q_{i}=0.1 I$, and $R_{i}=1$ for each $i=1,2,3,4$.

From the numerical simulation, we obtain the long-run average cost

$$
J(\varphi(0))=448.95 .
$$

We observe in the numerical simulation that the value in (27) remains unchanged under many different initial conditions $\varphi(0)$. This is a strong indication that (27) holds for all $\varphi_{i}(0) \in \mathbb{R}^{n}, i=1, \ldots, 4$, so that we can employ Theorem 2.1 to conclude that the simultaneous control system (19) is uniformly second moment stable under $\mathbf{f}_{s}=\{f, f, \ldots\}$. Equivalently, Theorem 2.1 assures that the trajectory $\Psi(k), k \geq 0$, is bounded and this behavior is corroborated by the simulation depicted in Fig. 1.

\section{Acknowledgements}

The first author thanks Prof. Enrique Zuazua, Scientific Director of BCAM - Basque Center for Applied Mathematics, for helpful discussions and suggestions. 


\section{REFERENCES}

[1] A. N. Vargas and J. B. R. do Val, "Average cost and stability of time-varying linear systems," IEEE Trans. Automat. Control, vol. 55, pp. 714-720, 2010 .

[2] — , "A controllability condition for the existence of average optimal stationary policies of linear stochastic systems," in Proc. European Control Conference, Budapest, Hungary, 2009, pp. $32-37$.

[3] — - "Average optimal stationary policies: convexity and convergence conditions in linear stochastic control systems," in Proc. 48th IEEE Conf. Decision Control and 28th Chinese Control Conference, Shangai, China, 2009, pp. 3388-3393.

[4] _ - "Minimum second moment state for the existence of average optimal stationary policies in linear stochastic systems," in Proc. American Control Conference, Baltimore, MD, USA, 2010, pp. 373-377.

[5] X. M. Yang, X. Q. Yang, and K. L. Teo, "A matrix trace inequality," J. Math. Anal. Appl., vol. 263, pp. 327-331, 2001.

[6] O. Hernández-Lerma and J. B. Lasserre, Discrete-Time Markov Control Processes: Basic Optimality Criteria. Springer-Verlag, New York, 1996.

[7] B. D. O. Anderson and J. B. Moore, Optimal Filtering. Prentice-Hall, Englewood Cliffs, N.J., 1979.
[8] F. Kozin, "A survey of stability of stochastic systems," Automatica, vol. 5, pp. 95-112, 1969.

[9] Y. Cho and J. Lam, "A computational method for simultaneous LQ optimal control design via piecewise constant output feedback," IEEE Trans. Systems Man Cybernetics Part B, vol. 31, pp. 836-842, 2001.

[10] G. D. Howitt and R. Luus, "Control of a collection of linear systems by linear state feedback control," Int. Journal Control, vol. 58, no. 1, pp. 79-96, 1993.

[11] R. A. Luke, P. Dorato, and C. T. Abdallah, "Linear-quadratic simultaneous performance design," in Proc. American Control Conf., 1997, pp. 3602-3605.

[12] J. Lavaei and A. G. Aghdam, "Simultaneous LQ control of a set of LTI systems using constrained generalized sampled-data hold functions," Automatica, vol. 43, no. 2, pp. 274-280, 2007.

[13] F. Saadatjooa, V. Derhami, and S. M. Karbassi, "Simultaneous control of linear systems by state feedback," Computers Math. Appl., vol. 58, no. 1, pp. 154-160, 2009.

[14] J. Wu and T. Lee, "Optimal static output feedback simultaneous regional pole placement," IEEE Trans. Systems Man Cybernetics Part B, vol. 35, pp. 881-893, 2005.

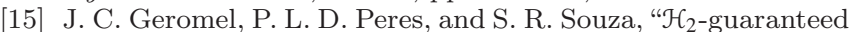
cost control for uncertain discrete-time linear systems," Int. Journal of Control, vol. 57, pp. 853-864, 1993. 